\title{
Laser Ablation of Polypropylene Films using Nanosecond, Picosecond, and Femtosecond Laser
}

\author{
Ik-Bu Sohn*, Young-Chul Noh, Young-Seop Kim, Do-Kyeong Ko, and Jongmin Lee \\ Precision Optics Lab., Advanced Photonics Research Institute, GIST, Oryong-dong 1, Buk-gu, \\ Gwangju 500-712, Republic of Korea \\ Young-Jin Choi \\ Daerung Packaging Industry, Sundong-ri 400, Chowol-eup, Gwangju city, Gyeonggi-do 464-864, \\ Republic of Korea
}

(Received March 3, 2008 : revised March 12, 2008)

\begin{abstract}
Precise micropatterning of polypropylene film, which is highly transparent in the wavelength range over $250 \mathrm{~nm}$ has been demonstrated by $355 \mathrm{~nm}$ nano/picosecond laser and $785 \mathrm{~nm}$ femtosecond laser. Increments of both the pulse energy and the shot number of pulses lead to cooccurrence of photochemical and thermal effects, demonstrated by the spatial expansion of rim on the surface of PP. The shapes of the laser-ablated polypropylene films were imaged by optical microscope and measured by a 3D optical measurement system. And, the ablation depth and width of polypropylene film ablated by femtosecond laser at various pulse energy and pulse number were characterized. Our results demonstrate that a femtosecond pulsed laser is an efficient tool for fabricating micropatterns of polypropylene films, where the micropatterns are specifically tailored in size, location and number easily controlled by laser processing conditions.
\end{abstract}

OCIS codes : $140.3390,350.3390$

\section{INTRODUCTION}

Polymer films play an important role in many industrial applications because of their highly adaptable properties and good thermal stability. In the past, several polymers have been studied extensively with respect to ns- and fs-laser ablation. The ablation threshold fluence for fs-laser ablation is reduced compared to the application of ns-laser pulses of the same wavelength. Laser ablation with femtosecond lasers is sufficiently promising for microfabrication of materials. For instance, a thermal affected zone was not formed in the ablated area even for metals [1]. Also, the high peak power of fs lasers could induce a multiphoton absorption to ablate transparent materials [2]. Similar results were also obtained in the case of polymer [3-6]. With the advance of femtosecond laser technology and polymer science, femtosecond laser-polymer interaction has been attracting more and more attention, and has recently directed polymer applications to fabricating microelectronic components and optical devices, according to their specific thermal, electrical, mechanical and chemical properties
[7]. It has also been demonstrated that transparent polymers can be processed with ultrashort laser pulses. This indicates that nonlinear absorption and/or incubation effects play an important role. The detailed ablation mechanism is still under discussion. It is also well known that femtosecond laser processing has great potential for ablation on polymers, two-photon polymerization, and direct writing [8-10].

Polypropylene (PP) has been widely used in the packaging industry because of its highly adaptable properties and good thermal stability, chemical resistance, physiological compatibility. In this paper, we compared a $785 \mathrm{~nm}$ femtosecond laser with $355 \mathrm{~nm}$ nanosecond and picosecond laser for ablating polypropylene film, and experimentally observed the ablation depths and widths for various pulse energies and pulse numbers.

\section{EXPERIMENTS AND RESULTS}

Oriented polypropylene (OPP) films with the thickness of $30 \mu \mathrm{m}$ were prepared. A nanosecond laser (8 ns, 
Quantel, Brilliant b) and a picosecond laser (30 ps, EKSPLA, PL2143) were used as a light source for ablation of OPP films. The beam has a wavelength of fundamental (1064 nm), second harmonics (532 nm), and third harmonics $(355 \mathrm{~nm})$, respectively. The laser pulses were guided into a microscope and focused by an objective lens with the focal length of $100 \mathrm{~mm}(\mathrm{NA}=0.055)$. The absorption spectrum of OPP film was measured by optical spectrometer. Figure 1 shows the OPP film spectrum. As shown in Fig. 1, the absorption spectrum is significantly decreased as the wavelength increases. When a pulsed UV laser irradiates the surface of an organic polymer, depending upon the incident wave-

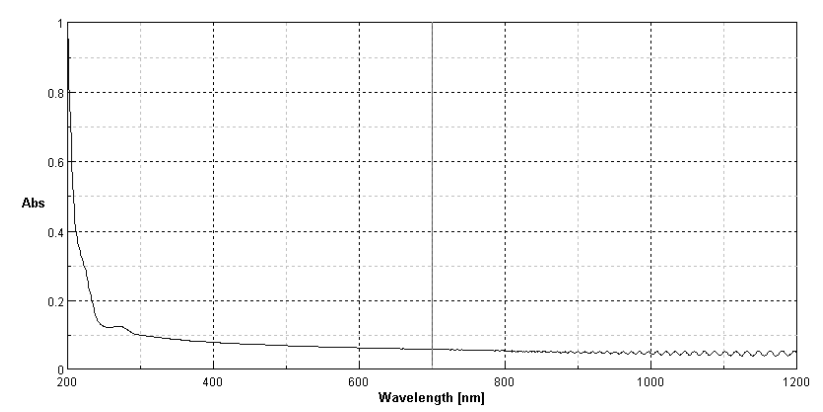

FIG. 1. Absorption spectrum of OPP film with a thickness of $30 \mu \mathrm{m}$. length, pulse number and pulse energy, a range from several hundred nanometers to tens of micron on the surface of the material could be ablated away with a geometry that is defined by the incident laser beam. Oriented polypropylene(OPP) films with a thickness of $30 \mu \mathrm{m}$, which run well over a wide range of temperatures and have good clarity and printability, were prepared for ablation. We compared a $355 \mathrm{~nm}$ ns and ps laser pulses with $532 \mathrm{~nm}$ and $1064 \mathrm{~nm}$ laser pulses for ablating polypropylene films. Compared to the $532 \mathrm{~nm}$ and $1064 \mathrm{~nm}$ ns and ps laser, the $355 \mathrm{~nm} \mathrm{~ns}$ and ps laser could etch PP film much faster and more precisely. As can be seen in Figs. 2 and 3, the OPP film was successfully ablated using $355 \mathrm{~nm}$ nanosecond laser and picosecond laser pulse with different fluences of $149 \mathrm{~J} / \mathrm{cm}^{2}, 298 \mathrm{~J} / \mathrm{cm}^{2}$, and $447 \mathrm{~J} / \mathrm{cm}^{2}$.

Femtosecond laser micromachining, materials processing and micro-structuring has become important in recent years for many fields including in micro-optics, micro-electronics, micro-biology and micro-chemistry. Laser ablation, because of its non-contact nature, allows the micromachining and surface patterning of materials with minimal mechanical and thermal deformation. These advantages lie in its ability to deposit energy into a material in a very short time period, before thermal diffusion can occur. As a result, the heat-affected zone, where melting and solidification can occur, is

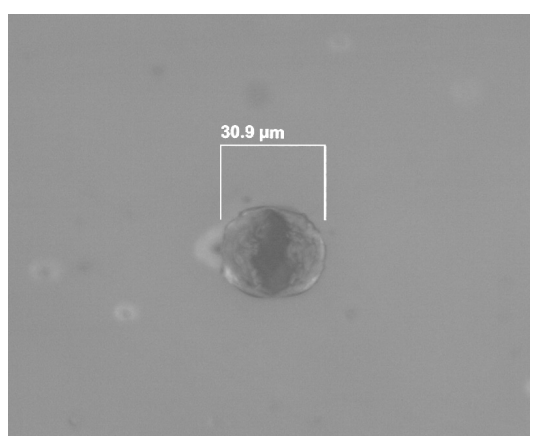

(a)

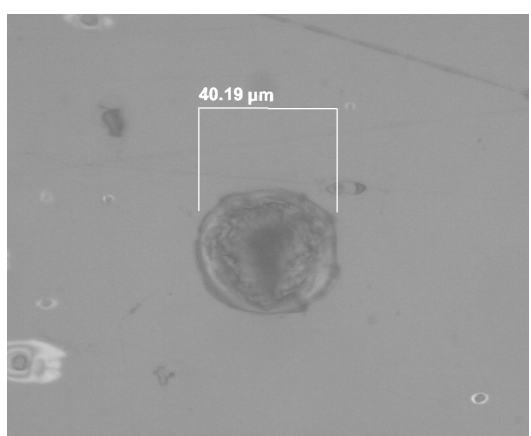

(b)

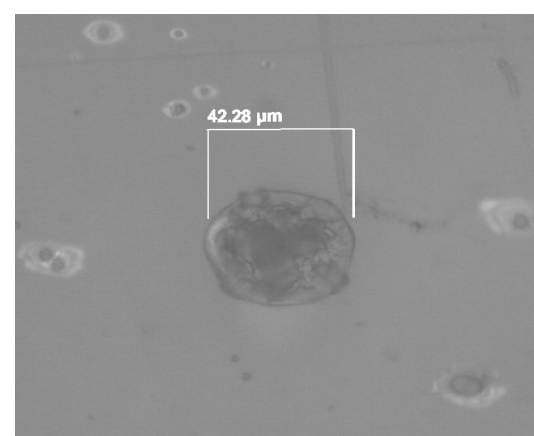

(c)

FIG. 2. Microscope image of OPP film ablated by a single $8 \mathrm{~ns}$ laser pulse with a pulse energy of (a) $149 \mathrm{~J} / \mathrm{cm}^{2}$, (b) $298 \mathrm{~J} / \mathrm{cm}^{2}$ and (c) $447 \mathrm{~J} / \mathrm{cm}^{2}$.

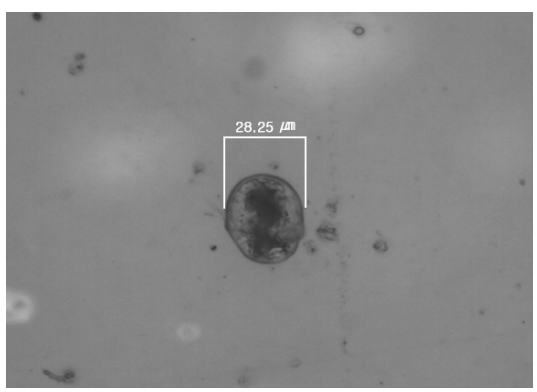

(a)

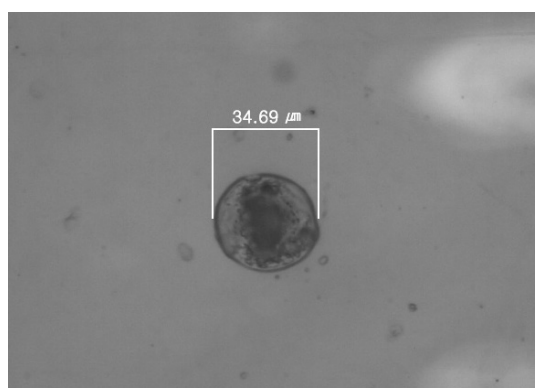

(b)

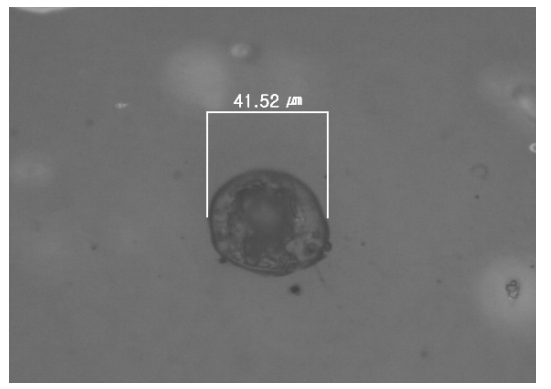

(c)

FIG. 3. Microscope image of OPP film ablated by a single 30 ps laser pulse with a pulse energy of (a) $149 \mathrm{~J} / \mathrm{cm}^{2}$, (b) $298 \mathrm{~J} / \mathrm{cm}^{2}$ and (c) $447 \mathrm{~J} / \mathrm{cm}^{2}$. 


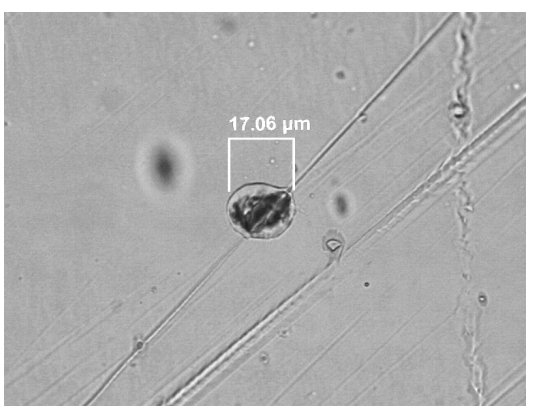

(a)

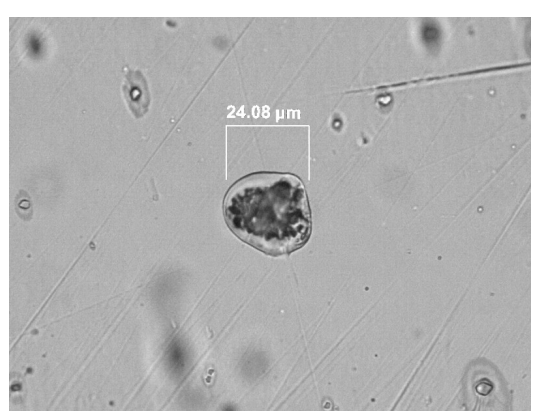

(b)

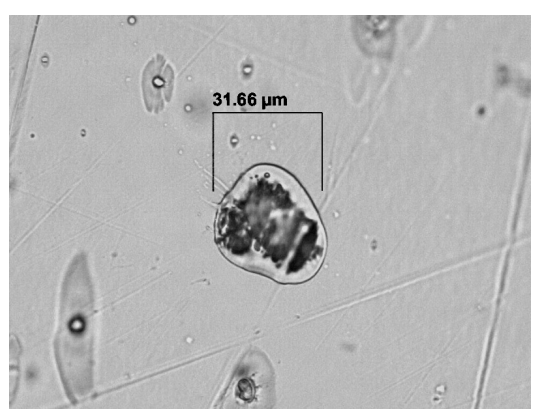

(c)

FIG. 4. Microscope image of OPP film ablated by a single 184 fs laser pulse with a pulse energy of (a) $44.5 \mathrm{~J} / \mathrm{cm}^{2}$, (b) $89 \mathrm{~J} / \mathrm{cm}^{2}$ and (c) $134 \mathrm{~J} / \mathrm{cm}^{2}$.

significantly reduced, leading to structured features that are smaller in size, have higher aspect ratios, and have greater spatial precision. In our experiment, we obtained good results for polypropylene films micropatterned by using a NIR femtosecond laser (Cyberlaser, IFRIT) with different fluences of $44.5 \mathrm{~J} / \mathrm{cm}^{2}, 89 \mathrm{~J} / \mathrm{cm}^{2}$, and $134 \mathrm{~J} / \mathrm{cm}^{2}$, as can be seen in Fig. 4. OPP films are ablated by femtosecond laser pulse at a lower fluence compared to nanosecond and picosecond laser pulse. The laser pulses were guided into a microscope and focused by an objective lens with the focal length of $100 \mathrm{~mm}(\mathrm{NA}=0.055)$. The average power of the laser beam is controlled by motorized attenuator, which consists of halfwave plate and polarizer. The sample is translated by a computer-controlled three-dimensional stage at a resolution of $100 \mathrm{~nm}$. The processes during the focused irradiation of femtosecond pulses were observed through a CCD camera mounted upon the microscope. For further investigation, the pulse number $\mathrm{N}$ was varied by using a synchronized pulse switching system, which consists of a synchronizing device and a Pockels-cell (Lasermetrics 5100EW $5 \mathrm{kHz}$ ).

In Fig. 5, the ablation width and depth versus pulse energy are shown for OPP film. For the OPP film, the ablation width of the crater increased with higher pulse energies but the depth was limited to near $23 \mu \mathrm{m}$ above the fluence of $134 \mathrm{~J} / \mathrm{cm}^{2}$. This means that it is not possible to drill through the polypropylene film with the thickness of $30 \mu \mathrm{m}$ using a single pulse fs laser pulse. The morphologies of the laser-patterned polypropylene films were characterized using an optical microscope and optical measurement system (NanoFocus).

When the pulse number was 1,2 , and 5 , the ablation width/depth were $30 / 15,35 / 20,40 / 30 \mu \mathrm{m}$, respectively. After the first pulse was deposited, the ablation depth was determined to follow a linear relationship with the successive pulses. We measured the ablation width and depth for various pulse numbers with an fs laser at the same pulse energy. With an increased number of applied laser pulses $(\mathrm{N}>5)$, the rim around the ablated craters was observed on OPP, which shows the crater of OPP

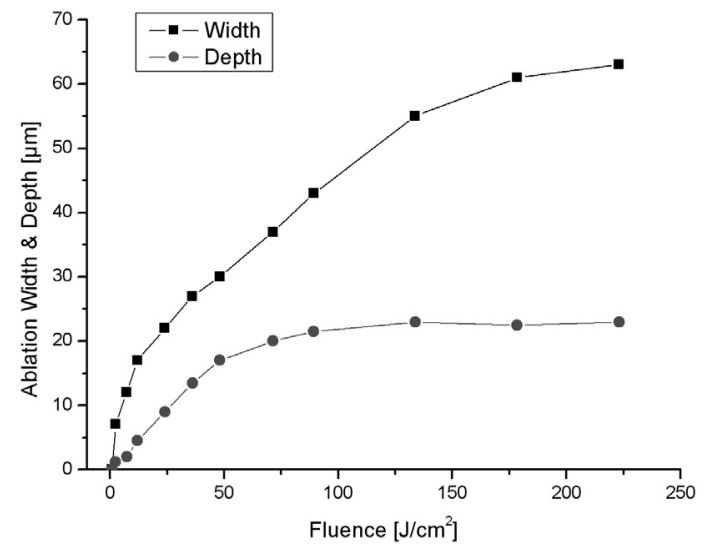

FIG. 5. Ablation width and depth versus fluence with a single femtosecond laser pulse.

irradiated by single and multi-shot laser with a fluence of $44.5 \mathrm{~J} / \mathrm{cm}^{2}$. The threshold was determined to be 7.12 $\mathrm{J} / \mathrm{cm}^{2}$ for $785 \mathrm{~nm}$ and 184 fs laser pulses with the input beam diameter of $5.6 \mathrm{~mm}$, the spot size of $16.9 \mu \mathrm{m}$, and the focal length of $100 \mathrm{~mm}(\mathrm{NA}=0.055)$. An ablation threshold is the minimum pulse energy per unit area needed to initiate the ablation process and is specific for each material. The threshold of OPP film was measured by patterning the surface with various pulse energies with a single pulse. An optical microscope was used to determine the ablation threshold, which was defined as the minimum pulse energy for an observable damage of the target surface. When the polypropylene is irradiated by a femtosecond laser with a pulse energy higher than the ablation threshold, the occurrence of ablation is mainly caused by the direct dissociation of molecules from the excited electronic states induced by the absorption of the laser photons. Meanwhile, the irradiation process co-presents a relaxation process that eventually converts photon energy into thermal energy. Therefore, different decomposition pathways will occur due to both photochemical and thermal effects. Formation of the rim is attributed to polymer melting and thereafter spreading across the 


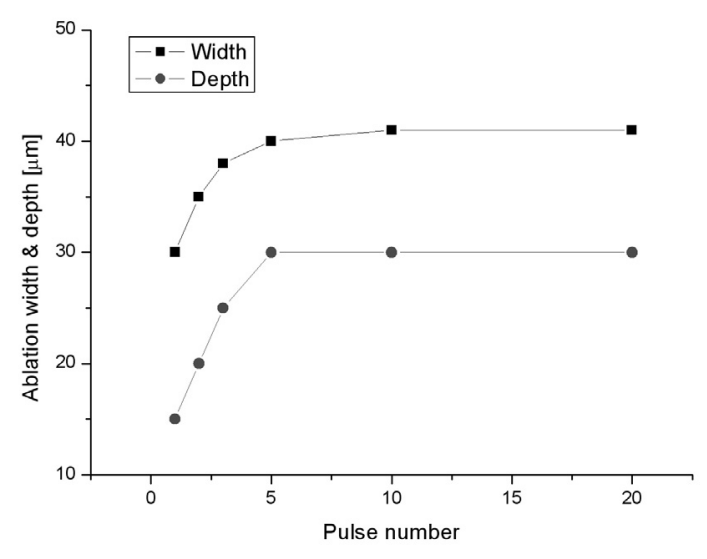

FIG. 6. Ablation width and depth versus pulse number with a femtosecond laser fluence of $44.5 \mathrm{~J} / \mathrm{cm}^{2}$.

crater. The driving force may result from surface tension due to a temperature gradient and a back pressure over the crater. It is therefore deducible that the spatial expansion of the rim is caused by growing mobility of the melt as a result of increasingly accumulated heat as the pulse energy and the number of pulses increase. Figure 6 shows the ablation width and depth of the crater versus the pulse number for linear polarized pulses. The pulse number was increased from $\mathrm{N}=1$ to $\mathrm{N}=20$ at the fixed fluence of $44.5 \mathrm{~J} / \mathrm{cm}^{2}$. The ablation width changes significantly during the first 5 laser pulses. For $\mathrm{N}>10$ the ablation widths are nearly constant. And, the ablation depth of the crater was increased with larger pulse numbers. We observed that the film with the depth of $30 \mu \mathrm{m}$ was penetrated by fs laser pulses above the pulse number of 5 . The image of the laserpatterned polypropylene films was characterized using a optical microscope and optical measurement system (NanoFocus). As a result, the ablation width and depth of micropatterns are adjusted by using the pulse energy, and number of micropatterns are optimally selected to obtain the desired gas transmission rate for the application to the breathable film, where the micropatterns are specifically tailored in size, location and number required.

\section{CONCLUSION}

We presented the ablation results of polypropylene films using $355 \mathrm{~nm}$ nano/picosecond laser and $785 \mathrm{~nm}$ femtosecond laser, and experimentally observed the ablation width and depth at various pulse energies and numbers. As pulse energy and the number of pulses increase, thermal effect and photochemical reaction coexist as mechanisms operative during polypropylene ablation process using a femtosecond laser. Oriented polypropylene films with the thickness of $30 \mu \mathrm{m}$ were micropatterned using femtosecond laser pulses. As a result, we have demonstrated that a femtosecond pulsed laser is an efficient tool for micropatterning of the polypropylene films.

\section{ACKNOWLEDGEMENTS}

This work was supported by the Ministry of Commerce, Industry and Energy (MOCIE) of Korea through the Industrial Technology Infrastructure Building Program.

*Corresponding author: ibson@icu.ac.kr

\section{REFERENCES}

[1] B. N. Chichkov, C. Momma, S. Nolte, F. von Alvensleben, A. Tunnermann, "Femtosecond, picosecond and nanosecond laser ablation of solids," Applied Physics A, 63, pp. 109-115, 1996.

[2] B. Wolff-Rottke, J. Ihlemann, H. Schmit, A. Scholl, "Influence of the laser-spot diameter on photo-ablation rates," Applied Physics A, 60, pp. 13-17, 1995.

[3] F. Beinhorn, J. Ihlemann, K. Luther, J. Troe, "Plasma effects in picosecond-femtosecond UV laser ablation of polymers", Applied Physics A, 79, pp. 869-873, 2004.

[4] M. Okoshi, N. Inoue, "Laser ablation of polymers using $395 \mathrm{~nm}$ and $790 \mathrm{~nm}$ femtosecond lasers," Applied Physics A, 79, pp. S395-S398, 2004.

[5] S. Baudach, J. Bonse, W. Kautek, "Ablation experiments on polyimide with femtosecond laser pulses," Applied Physics A, 69, pp. 748-750, 1999.

[6] Carlos A. Aguilar, Yi Lu, Samuel Mao, Shaochen Chen, "Direct micro-patterning of biodegradable polymers using ultraviolet and femtosecond lasers," Biomaterials, 26, pp. 7642-7649, 2005.

[7] Y. Feng, Z. Q. Liu, W. S. Yi, "Co-occurrence of photochemical and thermal effects during laser polymer ablation via a 248-nm excimer laser," Applied Surface Science, 156, pp. 177-182, 2000.

[8] O. Kondo, K. Yamasaki, S. Juodkazis, S. Matsuo, V. Mizeikis, H. Misawa, "Three-dimensional microfabrication by femtosecond pulses in dielectrics," Thin Solid Films, pp. 453-454, 550-556, 2004.

[9] J. Serbin, A. Ovsianikov, B. Chichkov, "Fabrication of woodpile structures by two-photon polymerization and investigation of their optical properties," Optics Express, 12(21), pp. 5221-5228, 2004.

[10] Shin Wook Yi, Seong Ku Lee, Hong Jin Kong, "Threedimensional micro-fabrication using two-photon absorption by femtosecond laser," Proceeding of SPIE, 5342, pp. 137-145, 2004. 